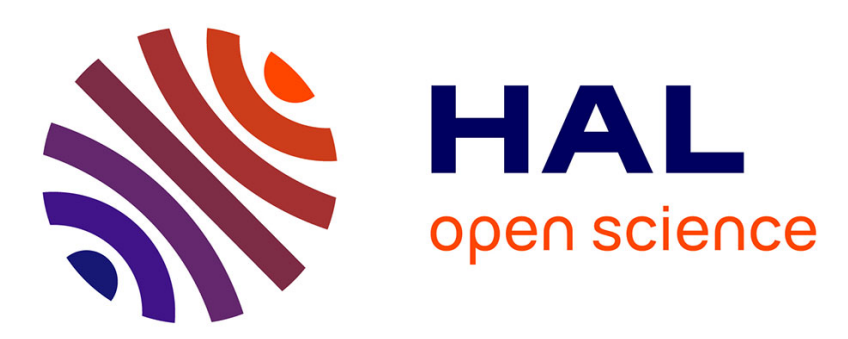

\title{
Culture des élites et culture(s) populaires(s) du règne d'Elisabeth à la Restauration : ponts et points de passage dans les productions visuelles et écrites
}

\author{
Luc Borot
}

\section{- To cite this version:}

Luc Borot. Culture des élites et culture(s) populaires(s) du règne d'Elisabeth à la Restauration: ponts et points de passage dans les productions visuelles et écrites. Bulletin de la Société d'Etudes Anglo-Américaines des XVIIème et XVIIIème siècles, 1998, 46 (1), pp.7-35. 10.3406/xvii.1998.1382 . halshs-01510972

\section{HAL Id: halshs-01510972 \\ https://shs.hal.science/halshs-01510972}

Submitted on 20 Apr 2017

HAL is a multi-disciplinary open access archive for the deposit and dissemination of scientific research documents, whether they are published or not. The documents may come from teaching and research institutions in France or abroad, or from public or private research centers.
L'archive ouverte pluridisciplinaire HAL, est destinée au dépôt et à la diffusion de documents scientifiques de niveau recherche, publiés ou non, émanant des établissements d'enseignement et de recherche français ou étrangers, des laboratoires publics ou privés. 


\section{Culture des élites et culture(s) populaires(s) du règne d'Elisabeth à} la Restauration : ponts et points de passage dans les productions visuelles et écrites

\section{Luc Borot}

\section{Citer ce document / Cite this document :}

Borot Luc. Culture des élites et culture(s) populaires(s) du règne d'Elisabeth à la Restauration : ponts et points de passage dans les productions visuelles et écrites. In: XVII-XVIII. Bulletin de la société d'études anglo-américaines des XVIle et XVIIle siècles. N46, 1998. pp. 7-34;

doi : 10.3406/xvii.1998.1382

http://www.persee.fr/doc/xvii_0291-3798_1998_num_46_1_1382

Document généré le 24/05/2016 


\section{CULTURE DES Élites ET CULTURE(S) POPUlAIRE(S) DU RÈGNE D'ÉLISABETH À LA RESTAURATION : PONTS ET POINTS DE PASSAGE DANS LES PRODUCTIONS VISUELLES ET ÉCRITES}

Après un examen approfondi de la question, il s'avère difficile, sinon impossible, de penser les stratifications ou circulations culturelles dans les termes extrêmes suggérés par le sujet de notre congrès: à l'époque moderne, il est impossible de ne pas tenir compte de l'émergence, parmi les consommateurs de culture les plus visés par le commerce de l'écrit, d'un public allant des yeomen aux marchands. Ce public aurait souvent refusé le qualificatif de "populaire" sans oser revendiquer celui d'aristocratique, espérant tout au plus un jour celui de gentle. Tous ces gens de la middle sort, ou middling sort, souvent perçus par leurs supérieurs sociaux comme a meddling sort, conquièrent la maîtrise de l'écrit et de l'imprimé pendant les décennies qui nous intéressent ici. Nous nous référerons plus souvent au couple notionnel retenu par Robert Muchembled, à savoir "culture populaire et culture des élites," sans pour autant adhérer à la totalité de son analyse. 'Aucune de ces catégories n'est d'ailleurs monolithique: il existe des cultures populaires, et une pluralité d'élites, sociales, religieuses ou intellectuelles, auxquelles des productions culturelles différentes peuvent s'adresser spécifiquement. Cependant, suivant une analyse de Peter Lake, on peut affirmer que si le tirage est important et si le profit est le motif dominant, alors on vise "le peuple."

Pour résumer le débat, selon Peter Burke et Robert Muchembled, la période moderne verrait, dans toute l'Europe, un couvercle répressif

1. Robert Muchembled, Culture populaire et culture des élites dans la France moderne (XVe-XVIIle siècles) (1978; Paris: Champs-Flammarion, 1991).

2. Peter Lake, "Deeds against Nature: Cheap Print, Protestantism and Murder in Early Seventeenth-Century England," Culture and Politics in Early Stuart England, ed. Kevin Sharpe and Peter Lake (Basingstoke: Macmillan, 1994) 257-58. 
s'abattre sur les pratiques festives populaires, à des rythmes variables selon les pays et les confessions, mais non moins efficacement. ${ }^{3}$ Margaret Spufford et Tessa Watt ont étudié la diffusion de la littérature de colportage et mis en lumière la difficulté d'identifier celle-ci comme un produit visant seulement le peuple. ${ }^{4}$ Pour atténuer quelque peu l'analyse de Burke sur la prise de contrôle des élites, Ronald Hutton étudie la circulation ascendante et descendante des pratiques, dont nous verrons des exemples, ${ }^{5}$ mais Keith Thomas, dans son article sur l'alphabétisation comme dans son livre sur le déclin de la magie, montre à quel point les représentations se transmettent principalement de haut en bas. ${ }^{6}$

Notre corpus est constitué de ballades, de pamphlets d'information ou canards, de newsbooks, de broadsides ou placards, couvrant une période d'une centaine d'années. Nous verrons successivement les problèmes de définition de la culture de l'imprimé, de sa réception et de sa diffusion. Nous verrons ensuite comment circulent dans les différentes aires sociales et culturelles des pratiques, des thèmes et des représentations, et nous examinerons, pour finir, la naissance de l'opinion publique nationale à travers la diffusion de périodiques d'information dans la première moitié du XVIIe siècle et surtout pendant la période révolutionnaire.

Entre le règne d'Élisabeth et la fin de la première révolution anglaise, on assiste à la conquête progressive de la médiation culturelle par l'imprimé, dans tous les milieux de la société anglaise. Cette conquête lente, qui fut le fait de militants évangéliques et d'auteurs de divertissements, procède par l'identification puis par la construction d'un public, et elle voit parallèlement se développer la perception de ce public comme un marché,

3. Peter Burke, Popular Culture in Early Modern Europe (London: Temple Smith, 1978) 205-86; Muchembled 223-380, surtout ch. 4 et 6.

4. Margaret Spufford, Small Books and Pleasant Histories: Popular Fiction and Its Readership in Seventeenth-Century England (London: Methuen, 1981); Tessa Watt, Cheap Print and Popular Piety, 1550-1640, Cambridge Studies in Early Modern History ser. (Cambridge: Cambridge UP, 1991).

5. Ronald Hutton, The Rise and Fall of Merry England: The Ritual Year 1400-1700 (1994; Oxford: Oxford UP, 1996) 66-67, 114.

6. Keith Thomas, Religion and the Decline of Magic: Studies in Popular Beliefs in Sixteenth-and Seventeenth-Century England (1971; Harmondsworth: Penguin, 1973) ch. 21-22, 756-800; "The Meaning of Literacy in Early Modern England," The Writtex Word, Literacy in Transition, ed. Gerd Baumann, Wolfson College Lectures, 1985 (Oxford: Clarendon, 1986) 97-131. 
dans une nébuleuse économique associant des libraires, des auteurs, des colporteurs et un public qui s'accoutume à l'usage de l'imprimé. ${ }^{7}$

Il serait trop simple de limiter la séparation entre culture populaire et culture des élites, en la résumant à une opposition entre une culture orale et une culture écrite ou imprimée. Des travaux récents font ressortir au contraire la présence active et indispensable d'innombrables médiateurs de l'écrit dans les milieux populaires, et nous montrent combien naturellement l'expérience de la lecture pouvait s'insérer dans les pratiques d'une culture orale.

Si la scolarisation était très faible, faute d'écoles, on met en question, grâce à une meilleure connaissance des procédures pédagogiques et de la diversité des stratégies d'accès aux compétences élémentaires de la lecture et de l'écriture, les évaluations pessimistes de David Cressy, fondées sur la fréquence relative des signatures et des marques lors des dépositions des témoins devant les Cours diocésaines de Norwich aux XVIe et XVIIe siècles. Selon David Cressy, synthétisé et corrigé par Margaret Spufford, on aurait un taux d'alphabétisation moyen, sur deux siècles, de $11 \%$ pour les femmes, de $15 \%$ pour les labourers, de $21 \%$ pour les husbandmen, de $56 \%$ pour les artisans et commerçants et de $65 \%$ pour les yeomen, la gentry étant créditée de $98 \%$ d'alphabétisation. ${ }^{8}$ On doit sérieusement nuancer ces chiffres: dans les day schools et dame schools des villages, on enseignait seulement la lecture des caractère gothiques et presque jamais l'écriture. La mémorisation jouait un rôle capital dans la pédagogie, comme l'attestent de surprenants témoignages cités par Keith Thomas, faisant état de dame schools tenues par des analphabètes qui connaissaient par cœur le contenu de leur matériel pédagogique sans savoir lire par ailleurs. ${ }^{9}$ L'apprentissage de l'écriture commençait seulement au bout d'un ou deux ans de scolarité, là où il y avait une école permanente, et on ne l'abordait souvent qu'avec le latin. ${ }^{10}$ Rares étaient les enfants dont les parents pouvaient économiquement se passer après l'âge de sept ou huit

7. Voir Cyprian. Blagden, "The English Stock of the Stationers' Company in the Time of the Stuarts," The Library 12.3 (September 1957): 167-86; Henry S. Bennett, English Books and Readers 1558-1603 (Cambridge: Cambridge UP, 1965) et English Books and Readers 1603-1640 (Cambridge: Cambridge UP, 1970); Watt 50-54.

8. David Cressy, "Levels of Literacy in England, 1530-1730," Historical Journal 20.1 (1977): 1-23; Spufford, "First Steps in Literacy: The Reading and Writing Experiences of the Humblest Seventeenth-Century Spiritual Autobiographers," Social History 4.3 (1979): 407-35 et Small Books 21.

9. Thomas, "Meaning of Literacy" 103.

10. Spufford, "First Steps" 411-12. 
ans, et qui pouvaient bénéficier de plus de trois mois de scolarité sporadique en tout et pour tout entre six et huit ans. ${ }^{11}$ Comme le souligne aussi Keith Thomas, on pouvait savoir lire l'imprimé, surtout la black letter, utilisée pour les proclamations officielles comme pour le cheap print, sans savoir lire une écriture manuscrite, car pour déchiffrer la secretary hand, il fallait savoir former les lettres. Les deux compétences n'étaient pas aussi liées que nous avons tendance à le penser. ${ }^{12}$

Les témoignages recueillis par Margaret Spufford, dans des autobiographies manuscrites d'hommes du peuple, révèlent de façon lumineuse l'importance des femmes, pourtant peu nombreuses à avoir été alphabétisées, dans la diffusion de la lecture et même de l'écriture dans la famille, la paroisse ou l'échoppe. La médiation culturelle des humbles, pour reprendre le terme de Margaret Spufford, manifeste le caractère fallacieux de la division entre un prétendu monde de l'écrit et un prétendu monde de l'oralité. La ballade ou le pamphlet acheté par un illettré et affiché au mur lui était lu par un membre de la communauté sachant lire, la maisonnée l'apprenait et pouvait transmettre aux voisins la connaissance du texte. De même, le fait d'apprendre à lire la Bible dans l'église inscrivait l'apprentissage de la lecture dans le cadre d'une culture orale, puisque apprendre à lire servait à donner à entendre la Parole à ceux qui ne pouvaient directement accéder à l'Écriture. ${ }^{13} \mathrm{Il}$ est donc possible d'imaginer une clientèle illettrée pour des productions imprimées. La Mopsa du Winter's Tale invitant son ami à lui offrir une ballade peut avoir été effectivement la cliente d'Autolycus: "Pray now, buy some: I love a ballad in print, alife, for then we are sure they are true. ${ }^{14} \mathrm{C}^{\prime}$ est en connaissant la nature de certains publics que les marchands concevaient leurs produits, pour les divertir ou pour les édifier, mais parfois aussi en jouant sur leurs instincts les moins nobles pour les divertir en prétendant les édifier. Il s'agissait à la fois de percevoir et de construire un public pour l'imprimé bon marché.

Pour rester dans le corpus shakespearien, que l'on pense à Autolycus, qui vend l'histoire du poisson apparu le mercredi 24 avril quarante mille

11. Spufford, "First Steps" 412, 414-15.

12. Thomas, "Meaning of Literacy" 99, 100, 107-10, 113-14.

13. Jonathan Barry, "Literacy and Literature: Reading and Writing in Historical Perspective," Popular Culture in England, c. 1500-1700, ed. Tim Harris, Themes in Focus ser. (Basingstoke: Macmillan, 1995) 83, 84.

14. William Shakespeare, The Winter's Tale, 1623, The Complete Works, ed. Stanley Wells and Gary Taylor (Oxford: Clarendon, 1988) 4.4.258-59, 1120. 
brasses au-dessus de l'eau en chantant la ballade contre les jeunes filles au cœur de pierre, au point qu'on crut que c'était une femme changée en poisson "for she would not exchange flesh with one that loved her" (Winter's Tale 4.4.273-79, 1120), ou que l'on pense à Trinculo dans The Tempest, rêvant au profit qu'il pourrait tirer en Angleterre de l'exhibition d'un monstre mi-homme mi-poisson comme Caliban $(2.2 .27-36,1178)$, on assiste à une parodie de la démarche commerciale qui pouvait conduire des imprimeurs d'ouvrages de dévotion à publier à vil prix des placards ou des canards au contenu non moins vil.

Au commencement auraient été le fait divers at le sensationnel. Au commencement aurait été le mépris du public le moins instruit, dont l'argent était désirable, quelque détestables que fussent ses goûts. Peutêtre... mais où commence, socialement parlant, le peuple pieux et (ou?) lettré? Où commence la populace assoiffée de sensations fortes? N'y avaitil que des journeymen dans le public des exécutions capitales? Roger Chartier définit le "peuple" culturel français dans la période moderne de façon négative, y rangeant ceux qui ne sont pas gens de robe: ni clercs, ni nobles, ni détenteurs de charges, ni gens de plume, ni médecins. ${ }^{15}$ Les marchands, comme les Jourdain de Molière, sont donc des gens du peuple par les origines, par l'éducation et par le goût. ${ }^{16}$ Si les Busy de Jonson, dans Bartholomew Fair, sont scandalisés par ce qu'ils voient à la foire, c'est qu'ils appartiennent au monde des godly. Des gens du même milieu sociologique, mais moins religieux, pouvaient trouver dans la même foire des occasions de divertissement honnête, ou de défoulement salutaire, ou encore d'encanaillement moins avouable.

Les profits de distinction, pour reprendre la formule de Pierre Bourdieu, rendent d'autant plus difficile la définition du "peuple" que cette notion n'est pas seulement socio-économique. S'il est un peuple revendiqué, comme celui des Niveleurs, même si certains venaient de la gentry, il est aussi un peuple fantasmé, dangereux comme les rogues de Robert Greene, ou idéalisé comme les bergers des pastorales. Revendiquer son appartenance au peuple quand on est marchand peut être d'autant plus problématique qu'on aurait bien du mal à faire accepter par ses voisins sa prétention à la gentility. Outre les publications religieuses, souvent plus

15. Roger Charticr, Lectures et lecteurs dans la France d'Ancien Régime (Paris: Seuil, 1987) 88; il reprend des catégories introduites par Daniel Roche.

16. Voir l'analyse de Chartier (58-59) sur la civilité et sur la distinction dans Le Bourgeois gentilhomme (1670) dans Molière, Euvres complètes, ed. Pierre-Aimé Touchard (Paris: Seuil, 1962) 506-41, surtout 3.3.517-20. 
durables, qui attirent tous les milieux et toutes les générations, l'imprimé le moins cher et le plus éphémère peut aussi tenter, aguicher bien des clients. L'alderman affichera-t-il dans la grande salle de sa demeure le placard représentant l'enfant difforme de Herne ou de Maidstone? En suivant Pierre Bourdieu, on aurait tendance à répondre par la négative, car ce bourgeois n'aurait aucun profit de distinction à en tirer auprès de ses égaux. Nous verrons plus loin que les auteurs et libraires savaient user du "profit de distinction" évangélique pour attirer ce public vers de pareils produits.

L'évolution du news print entre le sensationnalisme et le politique, dans les années 1560 à 1640, correspond à l'émergence d'une opinion publique, phénomène qui fait évoluer le premier journalisme vers un autre métier: il s'agira désormais pour ces auteurs de tenir un public de citizens informé de nouvelles pertinentes pour leurs intérêts économiques et politiques, et non plus d'édifier les simples par l'interprétation providentialiste ou patriotique d'un événement remarquable. Pour opérer ce saut qualitatif, il fallait que la nébuleuse professionnelle des imprimeurs, libraires et auteurs ait appris à traiter leur clientèle non plus comme un réceptacle passif, mais comme un marché à suivre et à observer.

Le public de l'imprimé réagit comme un marché. Parallèlement à la production lucrative de l'English Stock (Psautiers, catéchismes, almanachs, ouvrages de common law, primers), jalousement gérée à partir de 1603 par un syndicat fermé de professionnels londoniens tirant profit d'un marché captif, il reste bien des occasions de faire du profit avec des productions de masse. ${ }^{17} \mathrm{La}$ littérature de colportage en fait partie. Le colporteur sonde le terrain dans les campagnes et les bourgs, et, si la littérature de l'époque sur ce métier, par exemple les textes de John Taylor. n'est pas trop fantaisiste, ce professionnel est apprécié comme facteur. mais on peut aussi voir en lui un porteur de nouvelles pour sa clientèle autant qu'un informateur sur le public auprès de ses fournisseurs. ${ }^{18}$ Le travail de Tessa Watt sur la durée de vie des ballades pieuses montre combien la popularité d'un produit conditionnait sa survie, avec quelques exceptions toutefois, comme celle de cet imprimeur devant répondre de l'accusation d'impiété parce que dans le fonds hérité de ses ancêtres figurait une ballade prêtant à Adam et Ève des propos exceptionnellement

17. Blagden, "English Stock" 167-68.

18. John Taylor, The Carriers Cosmographie; or, A Briefe Relation of the Innes in and neere London STC 23740 (London: Griftin, 1637) sig.A2 $\mathrm{r}^{\circ}-\mathrm{A} 3 \mathrm{r}^{\circ}$. 
obscènes. Quand le syndicat des ballades se constitue en 1624, les ballades pieuses ont été remplacées dans la faveur du public évangélique par les psaumes métriques, et la ballade de divertissement domine le genre. ${ }^{19}$ L'institutionnalisation de la diffusion de ce corpus est peut-être l'indice d'une nouvelle division du marché et des genres, séparant la marchandise sacrée des denrées plus profanes. Cependant il est impossible de croire que les clientèles de ces produits aient pu être nettement différenciées. C'est aussi dans le sac du colporteur que les genres et les thèmes se croisent, ce qui correspond à la diversité du public rencontré par ce médiateur.

Les premières grandes synthèses, comme celle de Peter Burke ou celle de Robert Muchembled, faisaient apparaitre un sens de circulation des thèmes et des pratiques délibérément et uniformément descendant. Dans un processus de conquête et de conditionnement du peuple par les élites sociales et par les autorités confessionnelles, on aurait assisté en Europe, entre la Réforme et les Lumières, à l'imposition au peuple de pratiques et de modèles inspirés par les habitus des élites sociales, pour qu'au terme du processus, le modèle de la bourgeoisie s'impose comme norme. Si on peut faire assez souvent cette constatation, on est cependant obligé, à la lumière de travaux plus affinés, de nuancer considérablement cette vision, car il existe des moments et des lieux d'échange, et des renversements des échanges culturels entre ce qu'on appelle parfois aussi suivant une métaphore topique "le bas" et "le haut." La littérature n'est pas le lieu principal des rencontres. Ce lieu, ce moment privilégié des échanges en deux sens est la fête, qu'elle soit populaire, civique ou religieuse. S'il existe un corpus permettant d'observer au mieux ces circulations, c'est la littérature de colportage sous deux de ses formes: d'une part les récits de "jugements" divins et les récits de naissances humaines ou animales monstrueuses, et d'autre part les débuts versifiés du journalisme.

Grâce au travail récent de Ronald Hutton dans ses ouvrages The Rise and Fall of Merry England et The Stations of the Sun, on peut certes mesurer l'importance de la fête dans les stratégies de contrôle des élites urbaines sur les couches populaires, mais aussi et surtout la circulation des thèmes entre les fêtes populaires et les fêtes aristocratiques.

19. Pour l'affaire Gosson, voir Watt 74; sur l'évolution du genre, voir 55-73; sur le stock de 1624, voir 74-127. 
Les deux cas les plus évidents de circulation descendante étudiés par Ronald Hutton sont les Lords of Misrule et les morris dances. ${ }^{20}$ Nous n'examinerons ici que les premiers. Nous nous méfions de la théorie de Bakhtine, qui fait des personnages comme le Lord of Misrule des figures d'inversion témoignant du renversement subversif des hiérarchies par une culture populaire autonome pratiquant d'instinct le renversement des valeurs. En outre, une étude historique du Lord of Misrule laisse entrevoir qu'il peut être une confirmation de l'ordre: son règne est limité, ses pouvoirs, dans les faits, sont soumis à un contrôle parfois sourcilleux de l'autorité réelle, et s'il apparaît dans les fêtes populaires tudor, il vient des milieux raffinés de la cour et des Inns of Court, ce qui me semble limiter considérablement son caractère de subversion populaire. ${ }^{21}$

Le Lord of Misrule qui préside aux fêtes profanes de la Nativité n'est pas aussi subversif que le May King, c'est souvent un associé du master of the revels. La période de gloire des Lords of Misrule semble avoir été sous les règnes de Henri VIII et d'Édouard VI: sous ce dernier, le Lord officiel, Ferrers, mettait au point des divertissements de grand luxe, dont la fonction était d'amuser le jeune roi, mais aussi les Londoniens, car la Corporation avait aussi son Lord of Misrule, dont la complicité avec Ferrers éclatait dans des rencontres dignes des plus grandes fêtes de cour. ${ }^{22}$ C'est avec Marie Tudor que cette fonction disparut de la cour d'Angleterre, bien que la nouvelle reine continuât d'organiser des fêtes somptueuses pour la période des douze jours de Noël, pratique qui fut reprise par ses successeurs. Dans les demeures de la gentry, la pratique semble avoir duré plus longtemps, avec une renaissance sous Jacques Ier, sans doute dans les milieux anti-"puritains."

Des villes continuèrent aussi à organiser ou à tolérer des fêtes d'inversion, mais la peur de la populace mena à leur disparition progressive au tournant du XVIIe siècle. On ne peut donc dire uniformément que la circulation descendante des pratiques culturelles dans les années de la Réforme fut toujours un écrasement de la propension populaire à la réjouissance: le régime protestant du jeune Édouard en donne avec Ferrers l'exemple le plus frappant. C'est sous le règne d'Élisabeth que les

20. Sur l'origine aristocratique de la morris, voir Hutton, Rise and Fall 72, et pour un résumé de l'historiographie du phénomène et sur l'évolution de la danse, voir Hutton, The Stations of the Sun: A History of the Ritual Year in Britain (Oxford: Oxford UP, 1996) 262-76.

21. Hutton, Stations 109.

22. Hutton, Stations 105-06. 
réjouissances, qui avaient retrouvé leur vigueur sous Marie, commencèrent à marquer le pas, mais Ronald Hutton insiste sur la force qu'elles conservaient dans les années 1560 , notamment pour les fêtes de mai. ${ }^{23}$ Selon lui, les élites anglaises perdirent le goût du misrule festif après les vingt ans de misrule réel commençant en 1640, et, en 1680, John Aubrey pouvait voir dans les Lords of Misrule une chose du passé. ${ }^{24}$

Si la pratique des morris dances caractérise toujours pour nous les réjouissances anglaises, nous ne voyons plus de Lords of Misrule, plus de church ales, et si le personnage de Robin Hood est toujours une figure de la culture populaire moderne grâce au cinéma et à la télévision, nous ne l'associons plus à la culture des élites, alors qu'au XVIe siècle pendant environ une décennie, la célébration du hors-la-loi à la cour d'Angleterre figurait parmi les grands moments de l'année festive. C'est le cas le plus marquant de circulation ascendante d'un thème. ${ }^{25}$ Selon Ronald Hutton, le personnage populaire des pièces anonymes et des ballades, dont la présence serait attestée dès le XIIle siècle, est adopté par la cour du jeune Henri V.III en 1510, après être passé par les paroisses et par les demeures de la gentry, pour quitter la cour en tant que moment privilégié des fêtes de mai dans les années $1520{ }^{26}$ C'est lui qui fait le retour le plus notable lors de la restauration du Catholicisme sous Marie, mais son intérêt auprès des grands est éteint, et c'est dans les fêtes de paroisse qu'il revient à la mode. Après la Restauration de 1660, quand le marquis de Newcastle, soucieux de tenir le peuple dans les justes limites de sa sujétion, conseillait à Charles II de réactiver les Sunday sports et les morris dances, en insistant sur les personnages populaires de la geste de Robin Hood, ce n'était déjà plus aux fêtes de cour ou aux réjouissances villageoises qu'il pensait, mais à leurs incarnations populaires dans la morris, car c'est dans ce cadre qu'un personnage comme Maid Marian, toujours un travesti, a le plus longtemps survécu. ${ }^{27}$

23. Hutton, Rise and Fall 114-18.

24. Hutton, Stations 109.

25. Hutton, Rise and Fall 72, Stations 271-76.

26. Hutton, Stations 271, Rise and Fall 67. Hutton renvoie pour l'histoire de Robin Hood aux travaux de David Wiles, notamment The Early Plays of Robin Hood (Cambridge: Cambridge UP, 1981).

27. On nomme parfois ce texte "Advice" to Charles II, written and presented to the King in 1660" [Bodl. MS Clarendon 109], édité sous le titre "William Cavendish [A Treatise on Government]," A Catalogue of Letters and Other Historical Documents Exhibited in the Library at Welbeck, ed. S. A. Strong (London: John Murray, 1903) 227. Hutton, Stations 274-75. 
Pour revenir à l'imprimé, on peut aussi observer dans le journalisme à sensation une circulation des représentations et des mentalités, notamment dans les documents impliquant l'iconographie. Dans ces textes à gros tirage, bon marché, de mauvaise qualité, à l'écriture laborieuse ou inexistante, certains critiques voient une tentative d'inculturation du providentialisme protestant dans des mentalités populaires peu perméables à la nouveauté théologique. Helaine Razovsky y voit le signe d'un intérêt réel des couches populaires pour les questions d'herméneutique du livre de la Nature et du livre de la Parole, vue que nous ne partageons guère. Peter Lake, déjà cité, y voit une habile stratégie, parfois développée par des pasteurs comme Henry Goodcole, l'aumônier de Newgate, pour faire entrer dans la vie quotidienne des lecteurs ou des auditeurs populaires des récits de jugements divins, de crimes atroces et de réussites héroïques vécus par des hommes et des femmes du même milieu. ${ }^{28}$ Le lecteur pouvait aussi y trouver la réconfortante certitude de sa supériorité morale sur des criminels de même rang social, et il avait aussi le frisson malsain de lire des récits de crimes et de mutilations abominables. Cependant, contrairement à Helaine Razovsky, Peter Lake fait remarquer que le Protestantisme militant des narrateurs se manifeste surtout dans les récits de conversion et de repentance des criminels, où elles résultent toujours de la Providence et jamais du libre arbitre du personnage; pour le reste, précise-t-il, les miracles révélateurs comme celui dont nous allons bientôt parler, et la révélation du crime par le criminel endormi sont des éléments du merveilleux médiéval qu'on peut à bon droit hésiter à qualifier de proprement protestants. ${ }^{29}$ L'orthodoxie théologique tente donc d'utiliser ce moyen textuel de faire passer son message, mais, via l'iconographie qui accompagne les formes les plus simples de récits providentiels ou exemplaires, d'autres modèles "venus d'en haut" sont diffusés dans un public qui ne voit plus autant d'images dans les églises qu'avant les années $1530 .^{30}$

L'un des genres caractéristiques des années $1560-70$ est la ballade sur les naissances animales et humaines monstrueuses. La naissance d'un

28. Helaine Razovsky, "Popular Hermeneutics: Monstrous Children in English Renaissance Broadside Ballads," Early Modern Literary Studies 2.3 (1996): 1.1-34 [URL: http://purl.oclc.org./emls/02-3/razoball.html]; Lake 260-61, 267.

29. Lake $280,272-73$.

30. Tessa Watt explique que la multiplication des images pieuses de colportage dans les années 1620-30 correspond à un besoin populaire de visualiser la doctrine réformée, ce qui peut se faire dans le foyer, et non à l'église $(177,178-216)$. 


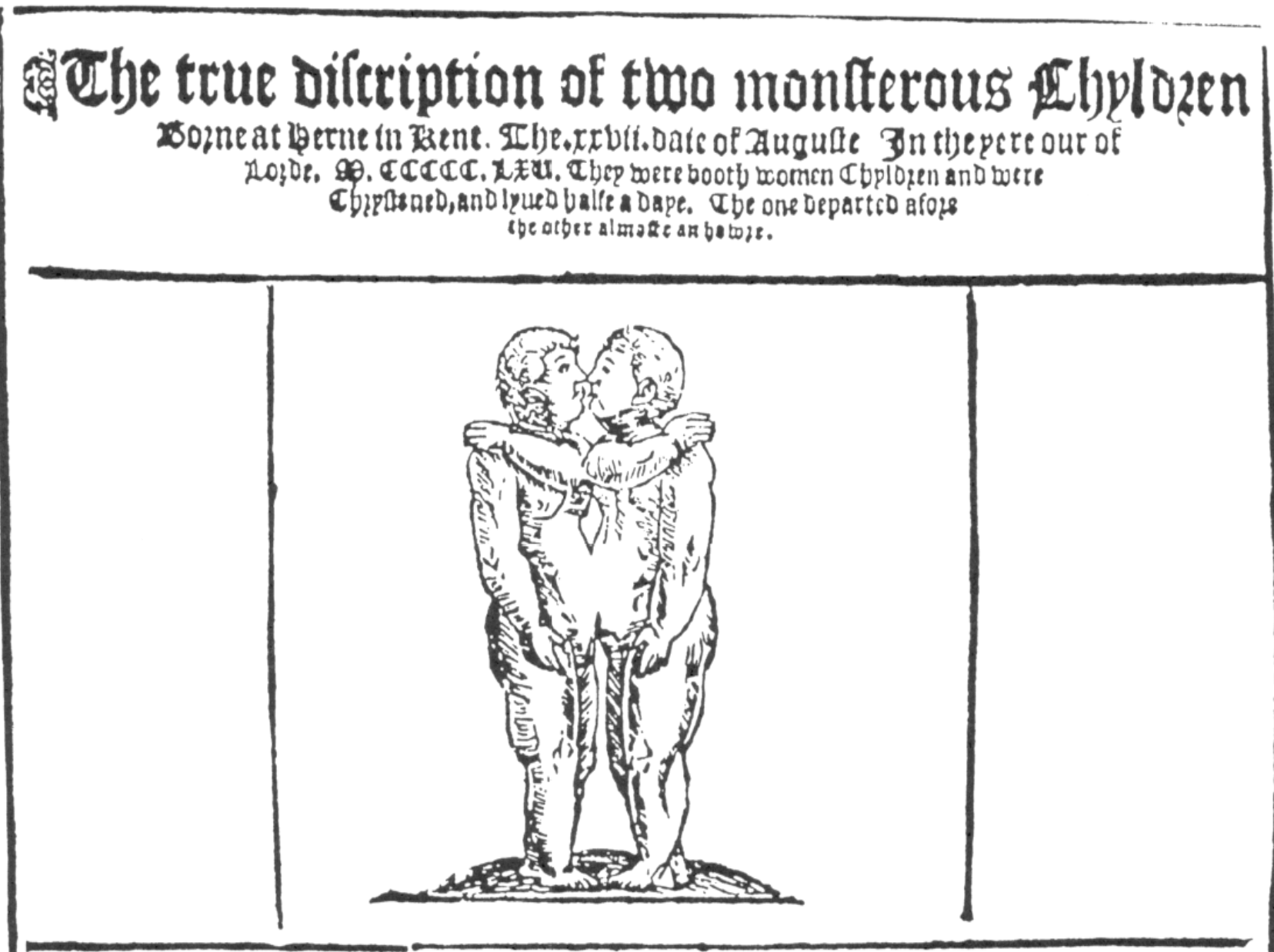

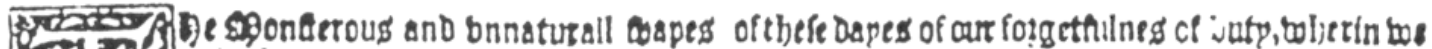

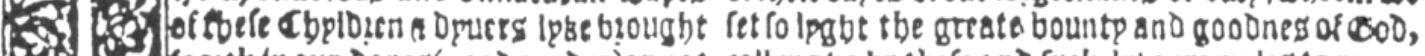

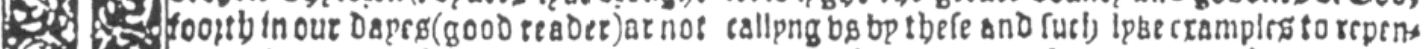

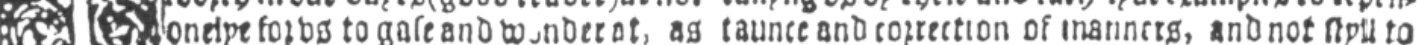

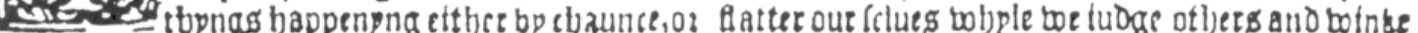

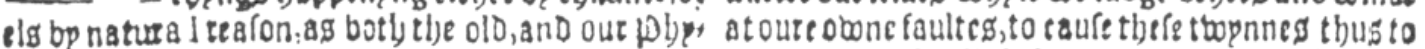

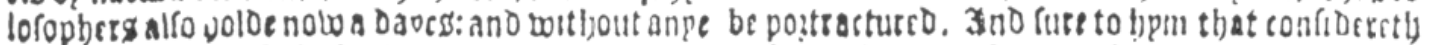
larther becDeto be bad tberto. or cls as our common as be ought to Do, the great Decap of bartp loue and

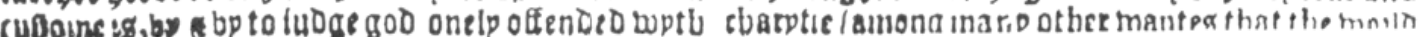

\section{The True Discription of Two Monsterous Chyldren}


enfant difforme ou de jumeaux siamois est une œuvre de la Providence. Les parents. souvent célibataires ou adultères, sont ainsi châtiés pour leur pratique du vice, mais les témoins-lecteurs-voyeurs des placards qui représentent ces monstres sont aussi concernés par le châtiment, car pour les auteurs ils ressemblent au moins autant aux monstres, ces pauvres créatures à la vie éphémère dont la difformité doit être lue comme une allégorie, qu'à leurs parents. Dans la description des sœurs siamoises de Herne dans le Kent, la moralisation est délibérée (Planche 1):

The monstrous and unnatural shapes of these children ... are not only for us to gaze and wonder at, as things happening either by chance, or else by natural reason, as both the old, and our philosophers also hold nowadays: and without any doubt ... as our common custom is, by and by to judge God only offended with the parents of the same, for some notorious vice or offence reigning alone in them. But they are lessons and schoolings for us all (as the word monster showeth) who daily offend as grievously as they do, whereby God Almighty, of his great mercy and long sufferance. admonisheth us by them to amendment of our lives, no less wicked, nay many times more, than the parents of such misformed be. ${ }^{31}$

Mais l'allégorisation ne l'est pas moins:

And sure to him that considereth as he ought to do, the great decay of hearty love and charity ... and hath viewed and beheld the two babes, the one as it were embracing the other, and leaning mouth to mouth, kissing (as you would say, one another), it might seem that God by them either upbraideth us for our false dissembling, and Judas' conditions and countenances, in friendly words covering Cain's thoughts and cogitations. or else by their semblance and example. exhort us to sincere amity and true friendship. . . . Neither let any man think this an observation over-curious, for as much as Christ himself hath by children taught us that unless we become like children, we shall not come in the kingdom of heaven.

Dans toutes ces ballades, la précision des dates, des lieux et des témoignages est frappante, mais en lisant les descriptions allégorisées des monstres, on a parfois peine à croire à la réalité de certaines naissances. $\mathrm{Si}$ nous regardons l'enfant de Maidstone (Planche 2, figure 3), en le comparant avec certains topoi iconographiques médiévaux, il fait penser

31. The True Discription of Two Monsterous Chyldren Borne at Herne in Kent (London: Rogers, 1565) STC 6774. Orthographe et ponctuation modernisées, grammaire conservée. L'analyse de Razovsky est très juste sur ce point précis. 
au sciapode, illustré dans la mapa mundi de la cathédrale de Hereford (Planche 2, figure 1), dans un manuscrit du voyage de Mandeville (Planche 2, figure 2) et dans la statuaire de nombreuses églises: le sciapode, cette créature qui s'abrite du soleil sous son unique et gigantesque pied. ${ }^{32}$ Dommage pour la crédibilité de l'auteur, qui devait être si satisfait de son allégorie du pied qui monte à la tête:

The leg so climbing to the head,

What meaneth it but this:

That some do seek not to be led,

But for to lead amiss.

And as this makes it more monstrous,

For foot to climb to head,

So those subjects be most vicious,

That refuse to be led. ${ }^{33}$

Le thème de l'évêque de mer, poisson connu de Gessler et de Paré, n'est pas évident dans l'image de ce poulpe géant (Planche 3, figure 1), mais les comparaisons que le texte établit avec le costume ecclésiastique peuvent créer un lien susceptible de suggérer une circulation du thème:

Here thou hast (gentle friend) the picture, shape and fashion of a fish strange and marvellous taken (as is said) in Holland, having on his fins hard scales in form much like the beggars' dishes, which in that country they were wont to wear in scoff and derision, his eyes like an owl, and mouthed as a popinjay, his tail red and four-cornered like to a priest's cap, which fish hath been seen and viewed of most nobles and peers of Flanders, who have plucked of his scales like to dishes, and keeps them for a show. ${ }^{34}$

La satire des mendiants, la queue en forme de barrette de prêtre, peuvent passer pour des éléments d'anticléricalisme populaire. Si l'on retourne l'image, on retrouve une forme qui n'est pas sans rappeler celle de

32. Nous devons ces exemples de sciapodes (comme l'évêque de mer étudié plus bas) à Éliane Cuvelier, Professeur émérite à l'Université de Paris-Sorbonne - Paris IV.

33. The Forme and Shape of a Monstrous Child, Borne at Maydstone in Kent the xxiv. of October. 1658 (London: Awdeley, 1568) STC 17194.

34. The Discription of a Rare or Rather Most Monstrous Fishe Taken on the East Cost of Holland the xvii. of November, Anno 1565 (London: Purfoot, 1566?). 


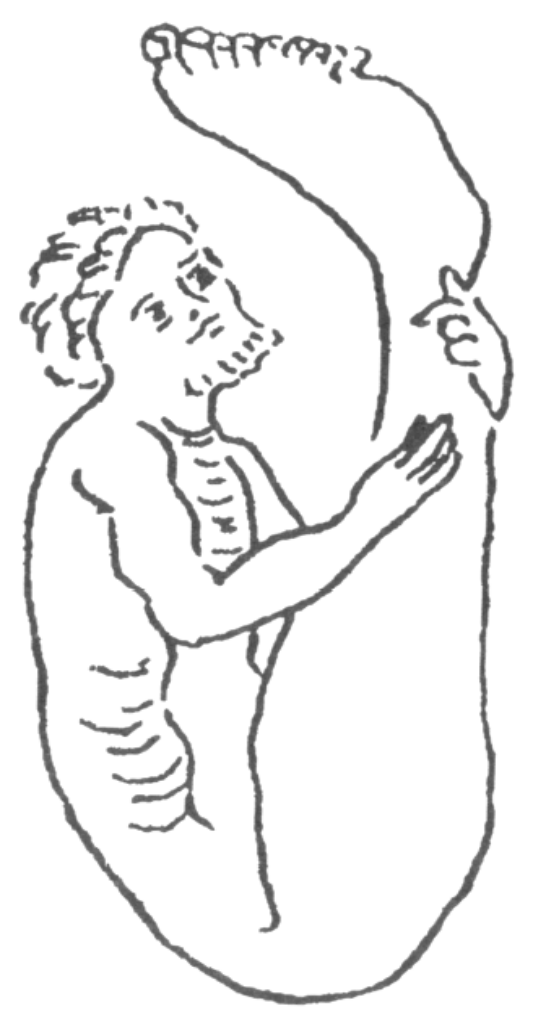

Figure 1

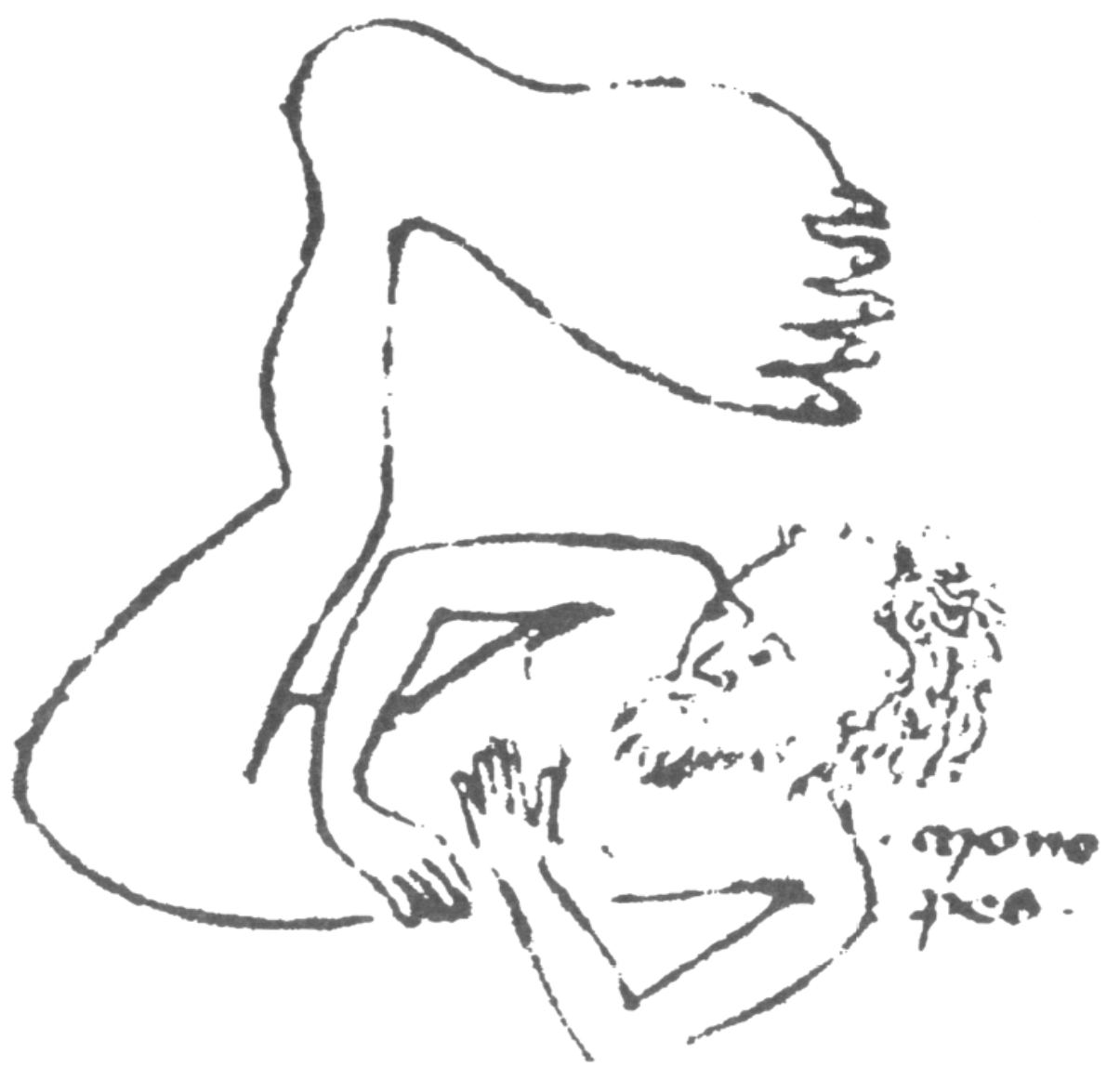

Figure 2

Planche 2 


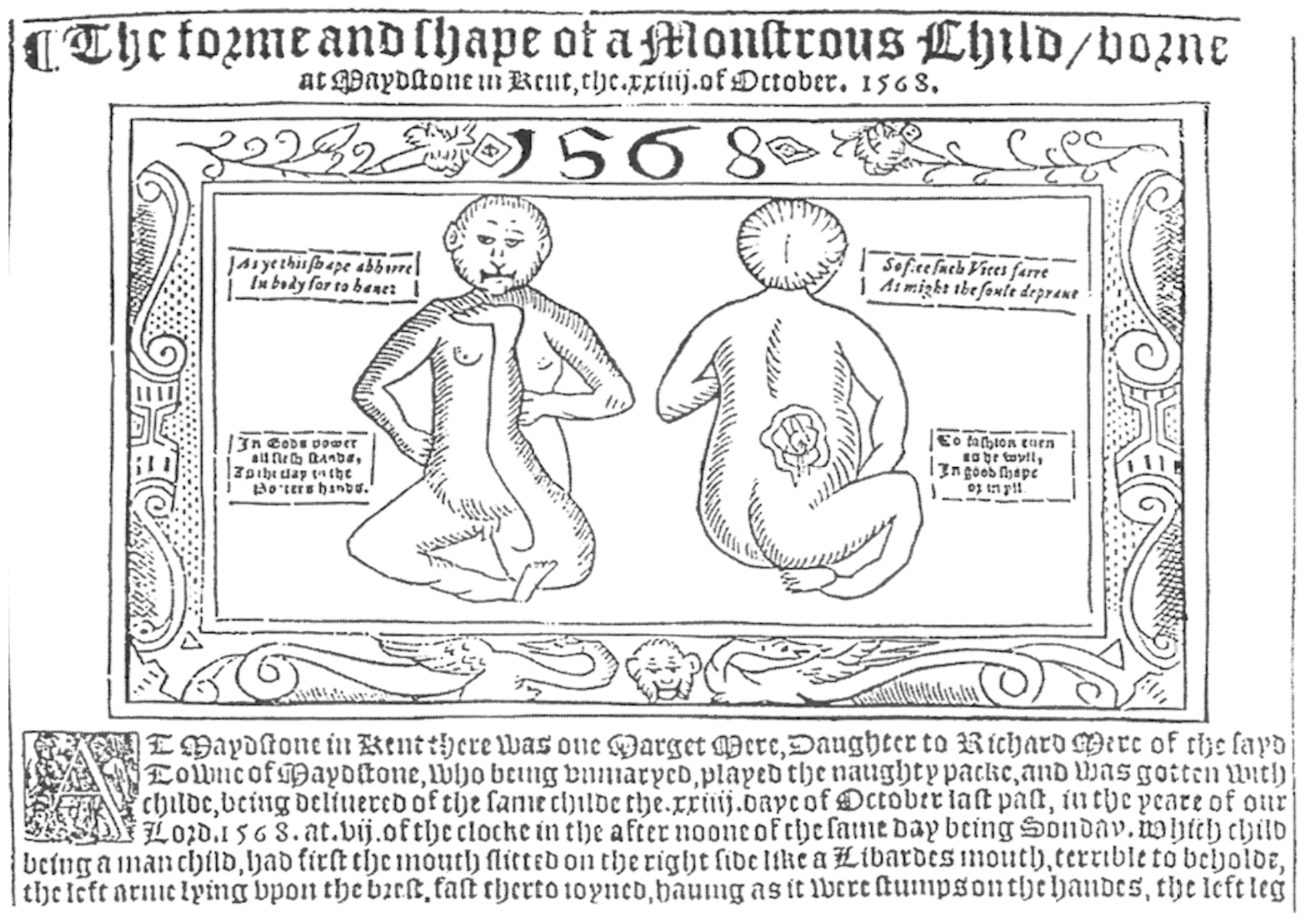

Figure 3 
l'évêque de mer (Planche 3, figures 2 et 3 ) ${ }^{35}$ Les premiers historiens de la nature dessinaient souvent sans modèle, d'après des récits et des descriptions plus ou moins précises. Si l'auteur de cette pièce de quatre sous est très précis sur les témoignages de véracité, il prend l'allégorisation providentialiste bien plus à la légère que ses confrères déjà cités:

And for the more credit hereof, ye shall understand that the 7th of December the said fish was brought to the city of Antwerp where it was openly showed and seen as well of Englishmen as other strangers. What this monster, with other uncouth sights seen of late do prognosticate and signify unto us, that I leave to thy conjecture (loving reader).... .

Ce placard est typiquement une pièce ironique: le ton en est clairement divertissant, l'image envahit la page, et les prétextes religieux sont traités en dernier, comme une pièce rapportée. Sur le mur de la chaumière, l'image sera décorative, la description est plaisante, mais le sérieux des descriptions d'enfants difformes est loin. L'esprit de Trinculo est déjà là, avec quarante ans d'avance.

C'est le moment de se demander pour quels types d'écrits on est en droit de parler de journalisme. Arundell Esdaile, qui a étudié ce qu'il appelle le "ballad journalism," y inclut tout récit d'événements mis en forme de ballade, ou publié sur un placard. ${ }^{36}$ Les événements politiques spectaculaires étaicnt fréquemment narrés sous forme de ballades, comme la répression des rébellions du Nord sous Élisabeth, ou les exécutions capitales des rebelles ou des missionnaires catholiques.

L'un des ballad journalists élisabéthains identifiables les plus prolifiques fut William Elderton. Il produisit des œuvres de circonstance sur l'exécution des rebelles, mais aussi des relations versifiées d'événements de cour exceptionnels, comme l'accident de chasse auquel Élisabeth échappa de peu en 1579. Un certain Thomas Appletree tira sans crier gare sur la Tamise un coup de fusil, alors que passait la péniche d'apparat de la reine. C'est l'occasion de démontrer le courage hors du commun de la souveraine, mais aussi sa mansuétude, car elle finit par gracier le manant sur l'échafaud. Même dans le danger la reine est grande,

35. Les deux images sont tirées de Jurgis Baltrusaitis, Réveils et prodiges. Le Gothique fantastique (Paris: Armand Colin, 1960) figures 17.A tirée de Conrad Gessner, Icones Animalium (Zürich, 1560) et 17.B tirée du Recueil de la diversité des habits (Paris, 1562).

36. Arundell Esdaile, "Autolycus' Pack; The Ballad Journalism of the XVIth Century," Quarterly Review 218.435 (April 1913): 372-91. 


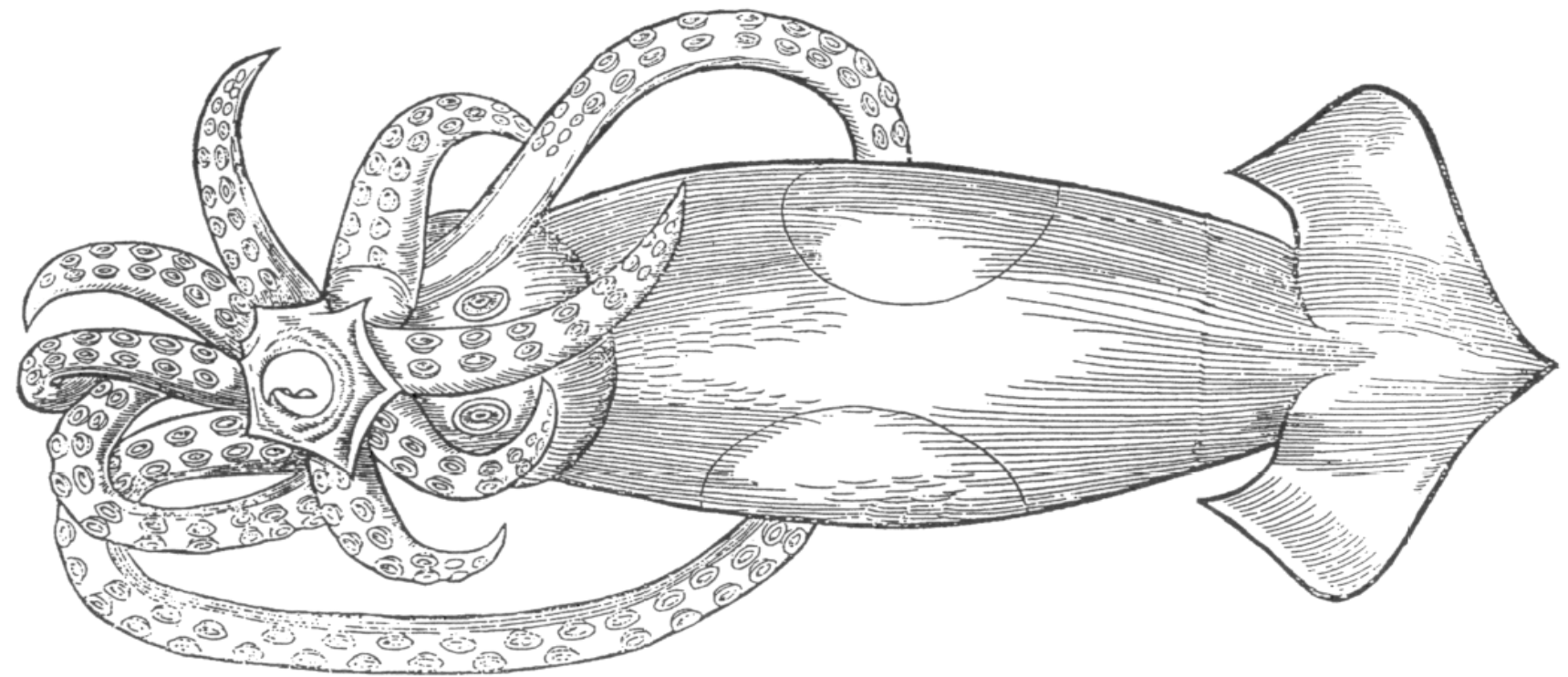

Figure 1

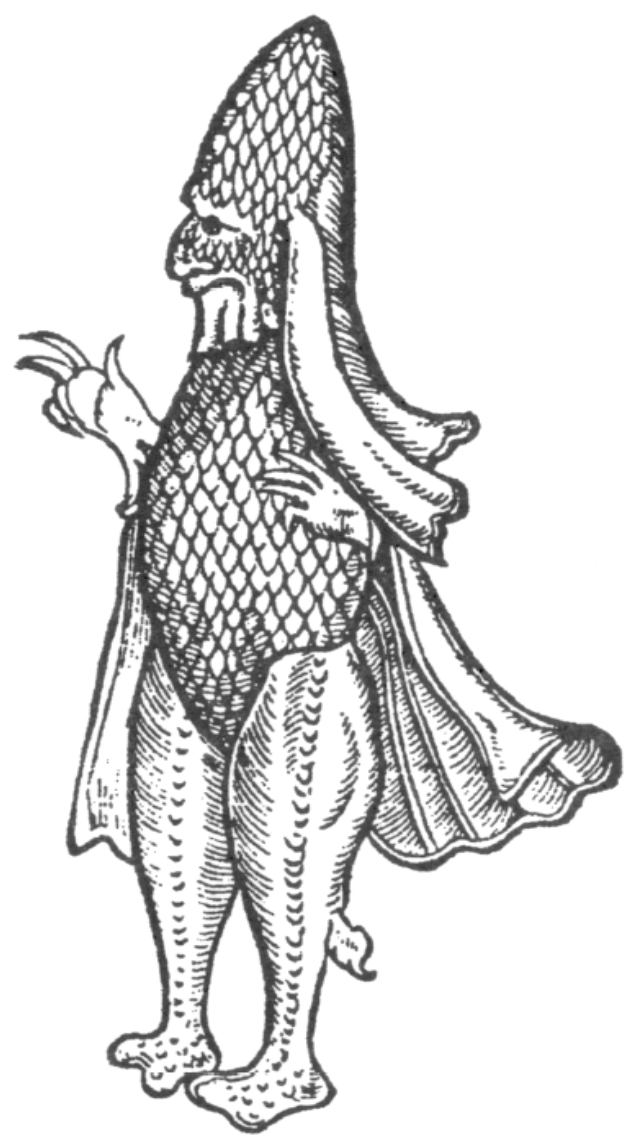

Figure 2

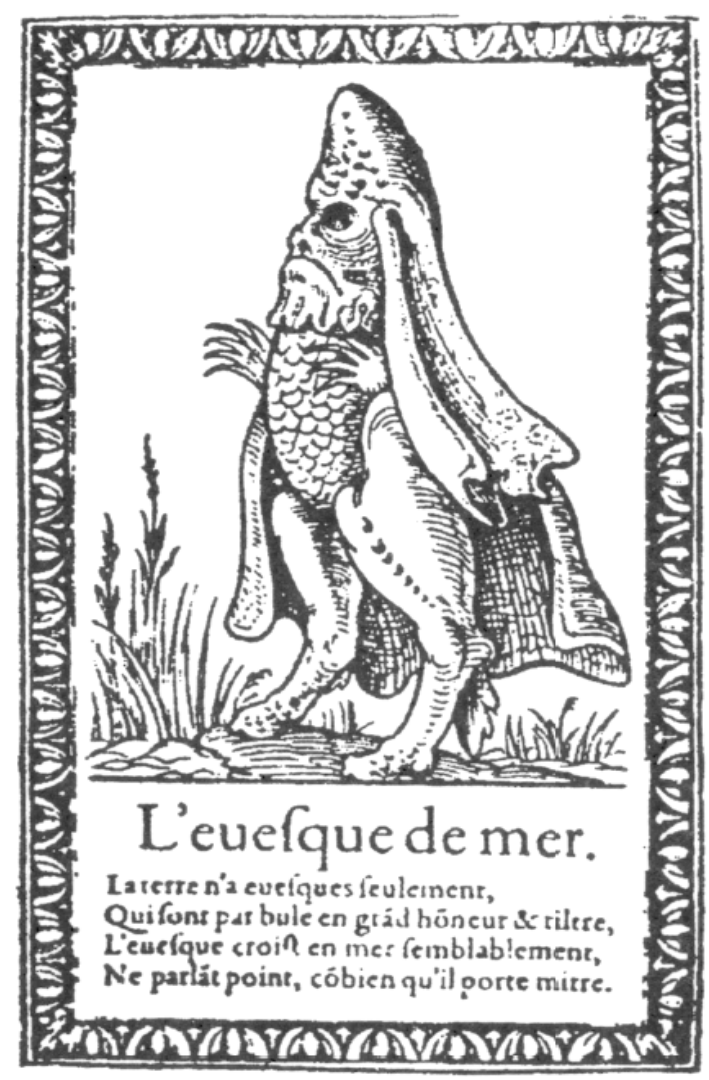

Figure 3

Planche 3 
c'est une femme mais elle est forte, elle est puissante mais miséricordieuse, même avec un domestique qui a involontairement mis sa vie en danger. La Providence veillait sur elle comme sur lui, et le versificateur appelle le peuple à l'action de grâce. La stratégie de narration ménage soigneusement le suspens, au début comme au moment de l'exécution. La mise en scène du journalisme à sensation est déjà en place; quatre strophes d'introduction, autant pour l'accident, autant pour le procès, trois sur l'échafaud, six sur la clémence de la reine et l'annonce de la nouvelle au condamné et aux spectateurs, et trois strophes de conclusion pour montrer l'effet de la miséricorde royale:

And then to hear the people shout, and see them clapped their hands

Who would have torn his flesh before. being in hangman's hands,

To see the goodness of her grace, to such great pity bent,

It made the stoniest heart of all, astonied to lament. ${ }^{37}$

Les procédés de sympathie, avec la reine d'abord, avec le condamné ensuite, puis d'empathie avec le public, créent des effets d'identification et de projection qui caractérisent toujours le journalisme à sensation.

Par exemple, dans le pamphlet de 1606 où la jeune fille muette retrouve la voix quand elle entre dans la maison où la cruelle Annis Dell a tué son frère et lui a coupé la langue quelques années auparavant, Dieu intervient pour accomplir le miracle, et les juges, appuyés par le témoignage d'un honnête tailleur du village, sont idéalement bons avec la victime et implacables avec les criminels. ${ }^{38}$ Dans un autre pamphlet sur le même événement, l'enfant est plus âgée, son frère mort est enfin nommé, la criminelle est l'aubergiste du village, mais le miracle de la muette retrouvant l'usage de la parole joue le même rôle. Pour attirer les clients, voire les acheteurs de l'autre pamphlet, le même libraire, Firebrand, ajoute l'histoire de la sorcière qu'on a brûlée le même jour à Hertford. ${ }^{39}$ On voit ici parfaitement le commerçant à l'œuvre: les maléfices de la sorcière ne nuiront en rien à ses bénéfices.

37. William Elderton, A Newe Ballade, Declaryng the Daungerous Shootyng of the Gunne at the Courte. To the Tune of Sicke and Sicke (London: White, 1579).

38. The Horrible Murther of a Young Boy of Three Yeres of Age whose Sister Had Her Tongue Cut out: and How It Pleased God to Reveal the Offendors, by Giving Speech to the Tongueles Childe. . . (London: Firebrand, 1606) STC 6552.

39. The Most Cruell and Bloody Murther Committed by an Innkeepers Wife, Called Annis Dell, and her Sonne George Dell. . . . (London: Firebrand, 1606) STC 6553. 
La critique sociale est assez violente dans le pamphlet sur l'assassinat sauvage du pasteur William Storre par l'un de ses paroissiens aisés. ${ }^{40}$ Après un office vespéral, ce pasteur était intervenu pour pacifier une dispute entre les freeholders de la paroisse et les Lords of the Manor, en prenant le parti des premiers mais en offrant de créer un groupe de réconciliation. L'un des fils de la gentry locale, un certain Cartwright, prit mal la chose et il commença a proférer publiquement des menaces de mort contre le pasteur et ses amis. Celui-ci s'en plaignit aux Juges de Paix, qui refusèrent de réprimander le jeune homme, mais s'engagèrent à le faire comparaitre aux prochaines quarter sessions. Quelques jours plus tard, Cartwright saisit un couteau et se livra avec rage au démembrement du pasteur, qui survécut une semaine à ses blessures et mourut en ayant édifié tous les témoins de son agonie. Les Juges de Paix, mettant sur le compte de l'emportement de la jeunesse la barbarie de Cartwright, le laissèrent libre sans le poursuivre.

Le narrateur est en totale sympathie avec le pasteur, le langage est celui du godly party, et le parti pris en faveur des freeholders suggère une opposition entre une population modeste puritaine et une gentry épiscopalienne, le jeune Cartwright ne cessant de qualifier le pasteur de priest comme pour le provoquer. Il est vrai aussi que c'est le narrateur qui prête ces mots au personnage. L'impression est toutefois renforcée par les listes de témoignages favorables au pasteur à la fin de l'ouvrage: paroissiens, propriétaires, autres pasteurs, et universitaires. Le narrateur tient ici le rôle du vengeur, que les juges n'ont pas assumé par solidarité sociologique, du moins la stratégie narrative le suggère-t-elle: il nomme le coupable, démontre sa culpabilité, insiste sur les motivations socioéconomiques, et dénonce l'injustice, mais le portrait du jeune homme est aussi une charge contre les péchés d'orgueil et de colère.

Ce journalisme à sensation peut donc se changer en journalisme engagé ou prendre parti dans des causes locales comme celle-ci, mais il va falloir plusieurs décennies pour que le coranto, gazette de nouvelles étrangères, laisse la place à des reportages et à des analyses plus sérieuses sur des événements qui ensanglantèrent l'ensemble de la Grande Bretagne et de l'Irlande.

40. The Manner of the Cruell Outragious Murther of William Storre Mast[er] of Arts, Minister, and Preacher at Market Raisin in the County of Lincoln (Oxford: Barnes, 1603). 
C'est sous Jacques Ier que se mettent en place un syndicat pour les corantos et newsbooks, et un autre pour les ballades. Le but des libraires et des imprimeurs est de tirer le plus de profit possible de ces marchés en vif développement, et celui du pouvoir est de contrôler le contenu potentiellement scabreux ou subversif de ces imprimés. Un public avide de nouvelles se manifeste avec tant d'ardeur à Londres que c'est rapidement lui qui devient une menace, car de la nouvelle à la fausse nouvelle, de celle-ci à la rumeur, et de la rumeur à la manipulation, le pas était aisé à franchir pour les opposants au régime, étrangers ou domestiques. La publication de nouvelles domestiques est donc interdite, et l'appétit de nouvelles doit se contenter d'informations étrangères; or comme dit le proverbe: "a beau mentir qui vient de loin." Dans le reste du pays le marché du coranto se développe, la poste fait circuler les newsbooks avec le courrier et, avec une brève interruption, le médium du périodique d'information se met en place dans les consommations de la société anglaise. Affiché, lu en privé, déclamé en public, envoyé par la poste, le journal est en train de prendre le relais du "canard sanglant."

L'enjeu de cette discussion pour le thème de notre congrès est capital: quand une opinion publique se constitue-t-elle, comment et à travers quoi? Habermas voit la sphère publique naître en Angleterre sous la Restauration dans une population aisée et désintéressée, mais c'est dans la guerre commerciale et dans la propagande protestante de la guerre de Trente Ans qu'elle voit le jour, quarante à cinquante ans plus tôt. ${ }^{41}$ Le moment de constitution de ce phénomène est essentiel: sans lui, il n'est pas possible de comprendre comment, grâce à la diffusion des nouvelles et des idées courantes sur les options politiques auxquelles la nation est confrontée, des couches sociales dont les intérêts sont différents peuvent s'allier pour lutter pour une cause commune, même si plusieurs causes communes sont en discussion comme au moment de la guerre civile.

Dès 1620, des feuilles de nouvelles imprimées en anglais à Amsterdam arrivent à Londres, et le 18 septembre 1621 , on cesse de les imprimer en

41. Jürgen Habermas, L'Espace public, 1962, trans. Marc B. de Launay (1978; Paris: Payot, 1993) 33, 43, critiqué par Joad Raymond, communication orale "News and the Public Sphere in the Seventeenth Century," colloque "The News, 1600-1800: New Approaches to Newspaper History in the Seventeenth and Eighteenth Centuries," U of London, Centre for English Studies, 10 May 1997. 
Hollande pour les produire directement en Angleterre. ${ }^{42}$ Entre 1632 et 1638 , les nouvelles seront interdites, avant l'explosion de la période révolutionnaire ${ }^{43}$ Étant données les limites de l'alphabétisation, le problème du lectorat se pose, mais l'apparition de ces nouvelles imprimées ne fait que diffuser une pratique bien plus ancienne, surtout dans les milieux du commerce, la lettre de nouvelles manuscrite, appelée letter of news, ou encore newsletter, que des cabinets et des scribes individuels produisaient pour une riche clientèle provinciale. Ces lettres circulaient dans les provinces, on les recopiait dans des journaux privés, et les Anglais commençaient au début du XVIIe à être friands de nouvelles. ${ }^{44}$

La vie londonienne autour de 1630 voyait chaque jour des centaines d'hommes s'assembler sur Paul's Walk pour échanger, lire et commenter les nouvelles. Toute une sociabilité de consommation journalistique se développait alors, au point de soulever l'ire satirique de Ben Jonson, qui consacra un masque et une comédie à ce phénomène et à ses héros, les libraires Nathaniel Butter et Nicholas Bourne, qui étaient au cœur du syndicat déjà cité. ${ }^{45}$ C'est contre le goût des journalistes pour le profit et contre la crédulité des lecteurs-clients que Jonson se déchaîne. Pour le Factor of news du masque News from the New World Discovered in the Moon, les nouvelles imprimées font du tort à ses affaires, car elles dévaluent les lettres. Il produit mille à mille deux cents lettres par semaine, les adapte à la religion de ses clients, et il a honte de voir des nouvelles imprimées côtoyer les histoires à dormir debout dont nous avons parlé plus haut:

[My news shall not be] trusted to your printed conundrums of the serpent in Sussex, or the witches bidding the devil to dinner at Derby:

42. Folke Dahl, "Amsterdam - Cradle of English Newspapers," The Library 4.3 (December 1949): 170. Joseph Frank, The Beginnings of the English Newspaper: 16201660 (Cambridge, MA: Harvard UP, 1961) ch. 1, 2-16.

43. Dahl 173-78; Frank, ch. 1, 14.

44. Richard Cust, "News and Politics in Early Seventeenth-Century England," 1986, The English Civil War, ed. Richard Cust and Ann Hughes, Arnold Readers in History ser. (London: Arnold, 1997) 234-37; Joad Raymond, The Invention of the Newspaper: English Newsbooks 1641-1649 (Oxford: Clarendon, 1996) 1-3.

45. Ben Jonson, Newes from the New World Discover'd in the Moone (joué en 1620; publié en 1640), ed. C. H. Herford, Percy and Evelyn Simpson, The Works of Ben Jonson, 11 vols. (Oxford: Clarendon, 1925-50) vol. 7, et Masques and Entertainments by Ben Jonson, ed. Henry Morley (London: Routledge, 1890); The Staple of News, 1626, ed. Anthony Parr, Revels Plays ser. (Manchester: Manchester UP, 1988). 
news, that when a man sends them down to the shires where they are said to be done. were never there to be found! ${ }^{46}$

À quoi le Chronicler répond en confirmant les propos de la Mopsa de Shakespeare:

It is the printing of them makes them news to a great many who will indeed believe nothing but what's in print. For those I do keep my Presses, and so many pens going to bring forth wholesome relations, which once in a score years, as the age grows forgetful, I print over again with a new date, and they are of excellent use. ${ }^{47}$

Le sentiment de submersion est aussi exprimé par le satiriste en prose Donald Lupton en 1632:

[These] are all conceits ordinarily, which their own idle brain, or busy fancies, upon the blocks in Paul's, or in their chambers invented. They have used this trade so long that now every one can say, it's even as true as a corranto, meaning that it's all false. . . Well, they are politic, not to be descried, for they are ashamed to put their names to their books. . . I I wish them either to write not at all, or less, or more true; the best news is when we hear no news. ${ }^{48}$

On peut pourtant se demander qui achète les newsbooks, et l'une des clés de ce problème est le prix: selon Joseph Frank, l'exemplaire coûtait 2 pence dans les années 1620 , et 3 pence dans les années 1630 , période de désaffection dont témoigne le texte de Lupton. Par comparaison, selon Richard Cust, Sir John Scudamore, du Herefordshire, payait à son news writer la somme de 20 livres par an ( 7 shillings par semaine) pour au moins une lettre par semaine, et un separate, manuscrit d'information, se vendait entre 6 pence et 2 shillings. ${ }^{49}$ Le prix de l'information manuscrite, considérée comme la plus fiable, était donc au moins trois fois plus élevé que celui de l'information imprimée, et quarante-deux fois plus si nous prenons le coût de la news letter de Scudamore comme référence pour le prix d'une information hebdomadaire générale spécialisée. L'information fiable était donc un produit de luxe. Le newsbook coûtait donc, si nous le comparons avec les ballades, deux ou trois fois plus, si le prix de la ballade est effectivement resté entre 1 penny et 1 penny et demi durant

46. Morley 243.

47. Morley 244.

48. Donald Lupton, London and the Countrey Carbanadoed and Quartered into Severall Characters (London, 1632) STC 16944, 141-43.

49. Cust 237-38. 
notre période. Pour un budget d'artisan salarié ou de domestique, c'est une grosse dépense; pour un petit artisan à son compte ou même un compagnon, c'est peut-être envisageable une ou deux fois par mois en période d'activité.

Quand les disputes du Long Parlement éclatent et que les parlementaires trouvent opportun de diffuser leurs idées pour en appeler à l'opinion londonienne (à coup sûr) et nationale (avec un peu de chance), ils contournent leur obligation de secret et laissent publier des versions édulcorées de leurs discours, ce qui laisse aussi la possibilité à leurs adversaires d'en publier des versions falsifiées. Malgré cet interdit, des libraires commencent à publier des comptes rendus des débats, des discussions entre citoyens londoniens et des bruits de couloirs des deux assemblées. Celles-ci réagissent en créant un comité chargé de réglementer ces publications. ${ }^{50} \mathrm{La}$ porte était ouverte aux hebdomadaires d'information et à la propagande qui s'en est vite emparée.

Le développement de la propagande dans les premiers mois de la guerre civile traite tous les milieux sociaux et culturels de la même façon: elle accélère la constitution d'une ou plusieurs opinions publiques divisées idéologiquement mais réunissant des Anglais de tous milieux et de tous lieux. La satire des pamphlétaires royalistes repliés à Oxford est d'une qualité littéraire et humoristique exceptionnelle, et le Parlement tarde à leur répondre, mais quand il le fait, c'est avec le talent de Marchamont Nedham, futur royaliste, futur thuriféraire de Cromwell, futur bénéficiaire d'une pension royale sous Charles II. Avec le journalisme politique naît la servilité journalistique comme la gutter press est née avec la presse dite d'information.

La mise en scène de l'information est un sujet trop complexe dès ses débuts, comme nous l'avons suggéré plus haut, pour le traiter ici en quelques lignes. Les newsbooks sont les porte-parole de nombreux courants d'opinion pendant les années 1640. Ils remplissent une fonction de service public pendant le procès du roi, et avant la répression cromwellienne de l'automne 1649, ils rendent à la liberté de la presse un témoignage qui, déjà, force le respect. Les périodiques royalistes clandestins sont aussi des véhicules remarquables du sentiment légitimiste dans ces mois terribles, et si Nedham, sentant tourner le vent, s'écarte de son déjà futur ancien camp royaliste durant l'hiver 1648-49, la verve et

50. Alan D. T. Cromartie, "The Printing of Parliamentary Speeches in November 1640-July 1642," Historical Journal 33.1 (1990): 23-44; Sheila Lambert, "The Beginning of Printing for the House of Commons, 1640-1642," The Library 3.1 (1981): 43-61. 
l'humour du désespoir montrent combien le triomphe républicain est fragile. $^{51}$

En regardant le corpus des pamphlets royalistes clandestins, on remarque combien l'argument que nous avons emprunté à Peter Lake au début de cet exposé est fragile: un pamphlet peut être imprimé sans aucun soin, sur du mauvais papier, ce qui nous permet de supputer qu'il n'était pas cher, tout en s'adressant à un public d'un niveau de culture littéraire très élevé. C'est le cas du savoureux Fairie Leveller, parodie anticromwellienne de Spenser, ou d'Hippanthrôpos (titre écrit en caractères grecs majuscules), pamphlet satirique sur la mort du Lord Mayor de Londres avec son cheval. ${ }^{52}$ Les références mythologiques sont trop nombreuses, et aussi trop elliptiques, pour s'adresser au public qui n'aurait lu que des ballades, des pamphlets édifiants et des murder pamphlets, ce qui nous fait penser que ces imprimés clandestins pouvaient être hors de prix, ou chercher à conquérir, par des prix très bas, des lecteurs qui, bien que n'ayant pas la culture littéraire permettant de saisir toutes les allusions, éprouvaient de la sympathie pour la cause royaliste.

Le dernier exemple sera l'Eikon Basilikè, moins pour son contenu que pour son icône (Planche 4). Rares sont les exemplaires du livre ayant survécu avec l'image du roi, le plus souvent déchirée pour servir d'icône murale ou portative. Le livre, publié dans les heures qui ont suivi l'exécution, a connu quarante-six éditions dans les douze premiers mois de son histoire. ${ }^{53} \mathrm{La}$ fréquence des représentations de Charles Ier dans la posture de cette gravure, dans des tapisseries au point de croix et des poteries, atteste de la popularité de cette image, et de sa très large diffusion. ${ }^{54}$ Cependant, le langage symbolique et allégorique de l'image relèvent de la culture de l'emblème, culture savante et raffinée. Les paroles

51. Frank et Raymond rendent parfaitement compte de cette évolution; on leur ajoutera Lois Potter, Secret Rites and Secret Writings: Royalist Literature, 1641-1660 (Cambridge: Cambridge UP, 1989) et James Loxley, Royalism and Poetry in the English Civil Wars: The Drawn Sword, Early Modern Literature in History ser. (Basingstoke: Macmillan, 1997).

52. The Faerie Leveller; or, King Charles His Leveller Descried \& Deciphered in Queene Elizabeths Dayes. . . (London, 1648) Thomason E.454 (23); IPPANTHRÔPOS; or, An Ironicall Expostulation with Death and Fate, for the Losse of The Late Lord Mayor of London. . . . (London, 1648) Thomason E.472 (18).

53. Edward Almack, ed., Eikon Basilike; or, The King's Book, 1649 (London: De la More $\mathrm{P}, 1904$ ) xii-xiii.

54. Les galeries de poterie et d'arts populaires du Fitzwilliam Museum (Cambridge) en possèdent plusieurs exemplaires. 


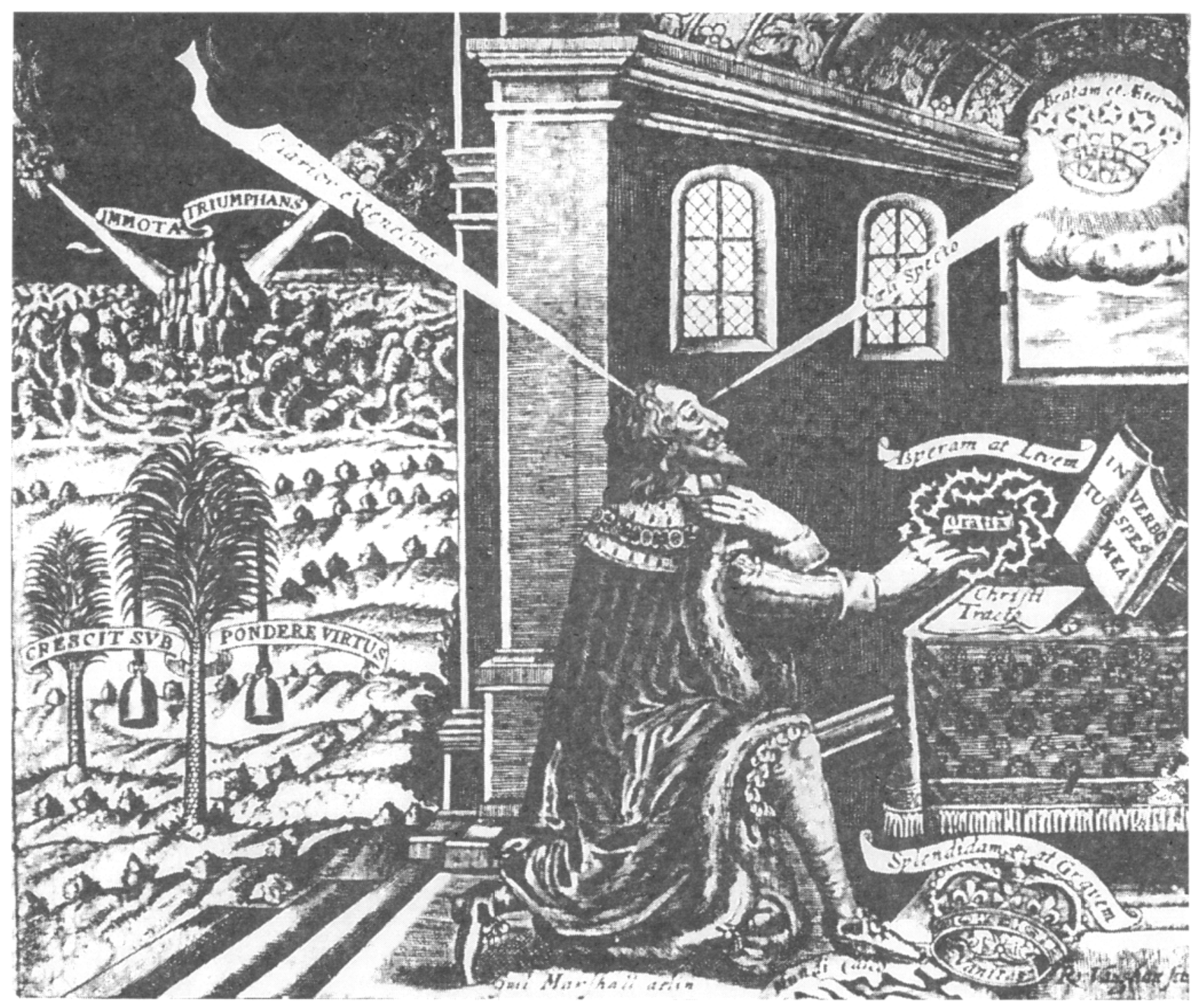

\section{Eikon Basilikè}


latines du roi dans la moitié droite de l'image et les allégories de la partie gauche ne la destinent en rien à devenir une image populaire. Le palmier, symbolisant la vertu, qui ne plie pas mais continue à pousser malgré les poids qui le retiennent est moins aisé à déchiffrer que la montagne stable au milieu des flots déchaînés, à l'arrière-plan. C'est le pathos de la posture, le destin du personnage et le profond attachement d'une partie du peuple à son roi qui expliquent que ce fruit exemplaire de la culture savante soit devenu une icône populaire. Le texte du roi ou de son auteur par procuration peut toucher, mais il n'est pas cans le style direct des publications visant un public populaire. La syntaxe et le vocabulaire des méditations sont en effet plus sophistiqués que dans les textes précédemment étudiés.

Le projet de l'Eikon Basilike peut donc apparaître comme le signe d'une absence de séparation entre des publics socialement et culturellement distincts. Comme les newsbooks, ce petit livre manifeste par son succès qu'une opinion publique est née ou en train de naître, rassemblant des sujets de tous milieux et de toutes les régions du royaume. L'erreur serait de croire que cette "sphère publique" créée entre autres par la circulation des nouvelles, se limite à Londres. Quels que soient les choix partisans des individus et leurs lieux de résidence, ils peuvent rencontrer des témoins des batailles, recevoir un pamphlet, assister à l'avancée des troupes, entendre des sermons dont la tonalité et les présupposés idéologiques et théologiques varient avec le flux et le reflux des armées. C'est tout le royaume qui est en désordre, c'est tout le royaume qui tente de dire ce désordre, en cherchant à savoir ce que cachent les massacres des desseins de Dieu et des hommes. ${ }^{55}$

Les années révolutionnaires apparaissent ici encore comme un grand renversement à bien des égards. Tout d'abord, l'opinion publique est façonnée par les journaux et par la propagande des parties en présence. En deuxième lieu, le pays est divisé suivant des axes idéologiques qui mettent en cause la structure hiérarchique et déférentielle et les allégeances territoriales traditionnellement en vigueur. Ensuite, l'explosion politique libère la presse d'opinion et le débat peut se renouveler avec des périodes de très large liberté jusqu'à 1649. Enfin, il n'y a aucune correspondance entre le milieu social d'un individu, son éducation et ses choix partisans à différents moments de la guerre civile et de la révolution.

55. Muchembled étudie le même phénomène à la fin de son ouvrage, à propos de l'utilisation de l'image du roi pour créer une psychologie collective de l'autorité (272-76). 
Si les Niveleurs et les Bêcheux, pour s'en tenir à ces deux groupes, expriment clairement des revendications dont l'origine réside dans les inégalités juridiques, constitutionnelles et sociales, ils ne sont pas les seuls porte-parole des milieux sociaux dont ils cherchent à défendre les intérêts et les droits. La révolution finit de faire éclater l'idéologie de la cohésion sociale naturelle, les publics s'entrecroisent, mais dans chaque camp, les élites veillent à ce que la hiérarchie sociale soit respectée dans la structure de décision.

Si le développement du news pamphlet et du newsbook peuvent donner le sentiment d'une diversification des voix proposant des opinions au public, l'échec des royalistes, accompagné de l'affirmation de la dictature militaire et confessionnelle de Cromwell et des siens, a imposé le modèle de la propagande littéraire et visuelle des élites jusque dans les milieux qui ne maîtrisaient pas nécessairement les codes de la littérature poétique ou emblématique. En même temps, l'abolition des fêtes de Noël et de Pâques, l'interdiction des réjouissances populaires par le nouveau régime confirment la tendance à la répression des couches populaires dans la pratique des divertissements traditionnels associés au cycle des saisons et aux temps liturgiques d'avant l'abolition de l'Église d'Angleterre. ${ }^{56}$

Enfin, pour résumer une année de recherche sur cette question, nous proposons quelques thèses. En premier lieu, la thèse de la grande répression des ardeurs festives populaires ne peut être totalement rejetée. Ensuite, si l'on ne peut parler d'une autonomie d'une culture populaire autonome et universellement définissable, il est en revanche possible de mettre en évidence des productions visant un lectorat ou un auditoire populaire et, dans la période révolutionnaire, pendant quelques années, des voix populaires authentiques. En dernier lieu, il existe un milieu sociologique qui est aussi bien visé par les publications lettrées que par les publications moins raffinées: il s'agit de ceux qu'on appelait the middling sort of people. C'est eux qui accédaient en grand nombre à la lecture dans cette période, c'est à eux que les newsbooks s'adressaient, ils pouvaient encore se laisser tenter par le cheap print adapté à leurs convictions religieuses et à leur désir de détente, et ils pouvaient aspirer à une participation civique effective dans le temps de leur génération. C'est dans cet espace traversé par des trajectoires sociales multiples que le

56. Le Niveleur Richard Overton s'oppose à ce mouvement dans The Baiting of the Great Bull of Bashan dès le printemps 1649; Revolutionary Prose of the English Civil War, ed. Howard Erskine-Hill and Graham Storey (Cambridge: Cambridge UP, 1983) 145-54. 
cheap print pouvait certainement trouver un public sans trop violer les prétentions au profit de distinction des candidats à l'ascension sociale.

Luc BOROT

Université Paul-Valéry - Montpellier III

Institut Universitaire de France 